\title{
AVALIAÇÃO DO CONCEITO DE CAPACIDADE DE CAMPO PARA UM LATOSSOLO AMARELO COESO DO ESTADO DA BAHIA(1)
}

\author{
A. O. AGUIAR NETTO(2), P. G. S. NACIF ${ }^{(3)}$ \& J . O. REZENDE ${ }^{(4)}$
}

\begin{abstract}
RESUMO
Por meio da morfologia do perfil e de análises físicas, tais como textura, densidade de partículas e do solo, distribuição do tamanho dos poros, retenção e armazenamento de água e capacidade de campo deter mi nada "in situ", avaliouse o comportamento de um Latossolo Amarelo, localizado em Cruz das Almas (BA), submetido à prática da irrigação. Os resultados obtidos evidenciaram que os horizontes AB e BA são bastante coesos, principalmente quando secos, e constituem forte impedimento mecânico ao crescimento radicular e à livre movimentação do ar e da água. Tendo em vista a presença de camadas coesas, o conceito clássico de capacidade de campo não se aplica ao solo em questão e, além disso, verificou-se que os teores de água no solo em equilíbrio com os potenciais de -10 e - $33 \mathrm{kPa}$, obtidos em laboratório com amostras deformadas, não representam devidamente o limite superior de água disponível nas condições de campo. Para calcular a faixa de água disponível, são indicados valores de potencial da água no solo, de acordo com os horizontes. Recomendam-se estudos específicos à origem da coesão observada, utilização de plantas ou implementos agrícolas capazes de romper a barreira física imposta pelos horizontes coesos.
\end{abstract}

Termos de indexação: propriedades físico-hídricas, coesão, tabuleiros costeiros.

(1) Trabalho recebido para publicação em outubro de 1997 e aprovado em fevereiro de 1999.

(2) Professor Adjunto do Departamento de Engenharia Agronômica, Universidade Federal de Sergipe. CEP $49100-000$ São Cristovão (SE). E-mail: aguiar@infonet.com.br.

(3) Professor Assistente do Departamento de Química Agrícola e Solos, EAUFBA. Doutorando em Solos e Nutrição de Plantas da Universidade Federal de Viçosa - UFV. CEP 363571-000. E-mail:pgabriel @solos.ufv.br.

(4) Professor Adjunto do Departamento de Química Agrícola e Solos, EAUFBA. CEP 44380-000 Cruz das Almas (BA). E-mail: joelito@ufba.br. 


\title{
SUMMARY: EVALUATION OF THE FIELD CAPACITY CONCEPT FOR A COMBINED LATOSOL FROM THE COASTING "TABULEIROS" IN THE STATE OF BAHIA, BRAZIL
}

\begin{abstract}
Through profilemorphology and physical analysis (texture, bulk and particledensities, poresizedistribution, water storageand retention, and field capacity determined "in situ"), the behavi or of a yel low Latosol located in Cruz das Almas (BA), Brazil, was studied when subjected to irrigation. The results made showed that the $A B$ and $B A$ horizons were hard (cohesion), mainly when dry, becoming a strong mechanic impediment fot root growth , aeration and water movement; due to the presence of the hard layer, the classic concept of field capacity was not applicable. Soil water contents in balance with potentials of -10 to $-33 \mathrm{kPa}$ and esti mated in thelaboratory using disturbed samples do not precisely represent the superior available water limit under field conditions. To cal cul ate the available water range, water potential values were chosen in accordance to the horizons. Specific studies about the origin the observed cohesion, the choice of plants and agricultural machinery to break the physical barrier imposed by hard layers and improvement of soil drainage are recommended.
\end{abstract}

Index terms: physical-hydric characteristics, cohesion.

\section{INTRODUÇÃO}

O conhecimento das inter-relações entre a água, o solo e a planta é essencial para uma eficiente explotação agrícola, porque a água necessária ao crescimento vegetal encontra-se principalmente no solo. O comportamento da água no solo depende fundamental mente de suas propriedades físicas.

O solo funciona como um reservatório de água para as plantas (Reichardt, 1988), porque, apesar de ser aberto para a atmosfera e para os horizontes mais profundos do perfil, ele retém água por meio de sua interação com a matriz. Com o propósito de quantificar o limite superior desse reservatório, Veihmeyer \& Hendrickson $(1931,1949)$ introduziram o conceito de capacidade de campo para caracterizar a quantidade deágua retida pel o sol o após o excesso ter sido drenado ea taxa de movimento descendente ter decrescido acentuadamente, o que, geral mente, ocorre dois ou três dias após uma chuva ou irrigação, em solos permeáveis e de estrutura e textura uniformes.

Os critérios para determinação da capacidade de campo são subjetivos, uma vez que o processo de redistribuição da água no solo é, na verdade, contínuo e não mostra interrupções abruptas ou níveis estáticos. Tal conceitoéimpreciso e nãoleva em conta uma série de fatores do solo responsáveis pela retenção e movimentação da água (Reichardt, 1975,1988).

Partindo da noção de que a capaci dade de campo é uma propriedade do solo e com a finalidade de simplificar sua determinação, foram estabel ecidas várias relações entre a umidade do solo na capacidade de campo e o potencial matricial da água no solo $\left(\Psi_{\mathrm{m}}\right)$, obtido em laboratório. J amison (1953) foi um dos primeiros a recomendar o uso de $\Psi_{\mathrm{m}}$ i gual a $-33 \mathrm{kPa}(-1 / 3 \mathrm{~atm})$ como limite superior da água disponível - valor que se generalizou ao ponto de quase se universalizar. Entretanto, esta é uma questão muito controvertida no meio científico, pois inúmeros autores, tais como: Salter \& Haworth (1961), Marcos (1971), Maclean \& Yager (1972), Borges (1979), Freire (1979), Ferreira \& Marcos (1983) e Medina \& Oliveira J r. (1987), estudaram e recomendaram valores de $\Psi_{m}$ entre -6 a $-50 \mathrm{kPa}$ ( $\Psi_{m}-6 / 100$ a $\left.-1 / 2 \mathrm{~atm}\right)$, para diferentes dasses desolo.

Por outro lado, pesquisadores contestam o estabelecimento de relações entre a capacidade de campo determinada "in situ" e os métodos de laboratório, porque ocorrem discrepâncias entreeles (Fernandes \& Sykes, 1968; Reichardt, 1975; Brunini et al., 1976) e não há fundamento teórico (Reichardt, 1988; Souza \& Reichardt, 1996).

Apesar dessas críticas à capacidade de campo, autores como o próprio Reichardt (1988) e Ahuja \& Nielsen (1990) reconhecem o valor desse conceito para fins práticos, desde que se compreenda o seu caráter dinâmico, variável no tempo e no espaço, dependente das propriedades hidráulicas de cada solo e dos limites da determinação, não se constituindo, portanto, em uma característica universal dos solos.

Nesse contexto, desenvolveu-se o presente trabal ho com o objetivo de estabel ecer relações entre a capacidade de campo determinada "in situ" e os dados obtidos em laboratório, para um Latossolo Amarelo coeso, representativo dos tabuleiros costeiros do nordeste brasileiro. 


\section{MATE RIAL E MÉTODOS}

O estudo foi realizado no município de Cruz das Almas ( $\mathrm{Ba})$, que possui as seguintes coordenadas geográficas: latitude $12^{\circ} 40^{\prime} \mathrm{S}$, longitude $39^{\circ} 06^{\prime} \mathrm{W}$ e altitude de 220 macima do nível do mar. Situada no planalto pré-litorâneo, Cruz das Almas apresenta clima tropical quente e úmido (Am), de acordo com a classificação de Köeppen.

O solo estudado pertence à classe dos Latossol os Amarelos, representativa dos tabuleir ros costei ros do nordeste brasileiro, e mostra, na área estudada, a seguinte descrição morfológica:

\section{Descrição morfológica}

Classificação: De acordo com o Sistema Brasileiro deClassificação de Sol os (EMBRAPA, 1999) o sol o foi dassificado como Latossol o Amarelo Coeso típico.

Localização: Iado direito da estrada que liga a Escola de Agronomia ao povoado Sapucaia, km 05.

Situação: Topo superior da encosta do tabuleiro, com declive de 1 a $2 \%$

Formação geológica e litologia: Terciário. Formação Capim Grosso. Sedimentos argiloarenosos.

Relevo: Local - plano; Regional - planoa suaveondulado

Altitude: $220 \mathrm{~m}$.

Pedregosidade: Não-pedregoso

Rochosidade: Não-rochoso

E rosão: Laminar ligeira

Uso Atual: Pastagem com capim Brachiaria decumbens

Descrito e Coletado por: Lucedino Paixão Ribeiro e Paulo Gabriel Soledade Nacif

Ap 0-6 cm, bruno-amarelado-escuro (10Y R 3/4, úmido); areia franca; blocos subangulares, fraca pequena a média, com raros grânulos; macio, muitofriável, ligeiramenteplástico, ligeiramente pegajoso; transição clara e plana.

$A_{2}$ 6-22 cm, bruno-amarelado-escuro (10YR 4/4 úmido); franco-arenoso; blocos subangulares, fraca pequena a média, com raros grânulos; macio, muito friável, ligeiramente pegajoso; transição clara e plana.

AB 22-45 cm, bruno-amarelado-escuro (10YR 4/4, úmido), franco-argilo-arenoso, maciço que se desfaz em fragmentos angulosos; duro, friável, plástico, ligei ramente pegajoso; transição clara e plana.

BA $45-73 \mathrm{~cm}$, bruno-amarelado (10YR 3/3, úmido), franco-argil o-arenoso, maciço que se desfaz em fragmentos angulosos; duro, friável, plástico, ligei ramente pegajoso; transição clara e plana.

$\mathrm{Bw}_{1} 73-123 \mathrm{~cm}$, bruno-amarelado (10YR 3/3 - úmido) franco-argilo-arenoso, forte pequena granular e blocos subangulares pequenos e médios; ligeiramente duro, friável, plástico, ligeiramente pegajoso; transição clara e plana.

$\mathrm{Bw}_{2}$ 123-180+cm, bruno-amarelado (10YR 3/3 úmido) franco argil oso, forte pequena granular e blocos subangulares pequenos e médios; ligeiramente duro, friável, plástico, ligeiramente pegajoso.

\section{Observações:}

- Porosi dade boa nos horizontes $A p, A_{2}, B w_{1}$ eBw 2 e deficiente nos horizontes $A B$ e BA.

- Atividade biológica moderada no horizonteAp, $\mathrm{A}_{2}$ e Bw $w_{2}$ e fraca no restante do perfil.

- Horizontes AB e BA com aspecto coeso.

Ressalta-se que os estudos físico-hídricos foram realizados até o horizonte $B_{w 1}$ (profundidade de $123 \mathrm{~cm}$ ), levando-se em conta o aspecto prático da irrigação.

As análises físicas foram realizadas em conformidadecom E MBRAPA (1979). Para cada caso específico, foram coletadas amostras de solo deformadas ou indeformadas nos diversos horizontes do perfil.

Para análise granulométrica, usou-se o método da pipeta. A densidade de partículas (Dp) foi obtida pelo método do picnômetro. Para determinar a densidade do solo (Ds), em cada horizonte do perfil do solo, foram coletadas quatro amostras de sol o com estrutura indeformada. Por meio da mesa detensão, utilizando as mesmas amostras col etadas para Ds, determinou-sea distribuição do tamanho dos poros.

A retenção da água no solo foi medida por meio do aparel ho extrator de umidade idealizado por Richards (1949). As amostras de terra fina seca ao ar, previamente saturadas, foram submetidas aos potenciais matriciais da água no solo de -4, -10, -33, $-100,-300,-900$, e-1.500 kPa, em duplicata. As curvas de retenção obtidas para cada horizonte foram ajustadas ao model o de van Genuchten (1980), por meio do programa CURVARET (Dourado N eto et al., 1990):

$$
\theta=\theta_{\mathrm{r}}+\frac{\left(\theta_{\mathrm{S}}-\theta_{\mathrm{r}}\right)}{\left[1+\left(\mathrm{a} \psi_{\mathrm{m}}\right)\right]^{\mathrm{b}}}
$$

em que:

$\theta=$ umi dade do solo, $\mathrm{m}^{3} \mathrm{~m}^{-3} ; \theta_{\mathrm{r}}=$ umidaderesidual do solo, $\mathrm{m}^{3} \mathrm{~m}-3 ; \theta_{\mathrm{s}}=$ umidade de saturação do solo, $\mathrm{m}^{3} \mathrm{~m}^{-3} ; \mathrm{a}, \mathrm{n}$ e b $=$ coeficientes empíricos; $\psi_{\mathrm{m}}=$ potencial matricial da água no solo, $\mathrm{kPa}$. 
Para determinar a capaci dade de campo "in situ", selecionou-se uma área próxima ao perfil do solo estudado, onde foram construídos diques de terra, recobertos com plástico, para possi bilitar a completa saturação de um volume de solo $(2,0 \times 1,0 \times 1,23 \mathrm{~m})$ e evitar a evaporação da água. Após a saturação do sol o, ea interval os detempo de $24 \mathrm{~h}$, duranteseis dias, col etaram-se amostras, em triplicatas, por meio de tradagens em cada horizonte, para fins de determinação da umi dade do solo, pelo método da estufa.

\section{RESULTADOSE DISCUSSÃO}

No quadro 1, encontram-se os resultados das análises físicas do solo. As classes texturais variaram de areia franca, no horizonte superficial, atéfrancoargilo-arenoso, no horizonte $\mathrm{B}_{\mathrm{w} 1}$. $\mathrm{O}$ solo estudado apresenta valores de densidade de partículas bem próximos da média $\left(2,65 \mathrm{~kg} \mathrm{dm}^{-3}\right)$ encontrada para a maioria dos solos minerais com predominância de caulinita equartzo (Kiehl, 1979; Oliveira et al., 1992). Quanto à densidade do solo, nota-se que os dados decrescem com a profundidade do solo, em decorrência do aumento de argila e diminuição da areia do horizonte A para o B.

$\mathrm{Na}$ análise da distribuição dos poros ao longo do perfil, verifica-se que a porosidade total é menor no horizonte $\mathrm{A}$ e na transição deste para o B, exatamente onde os valores da densidade do solo são mais elevados, indicando a ocorrência de compacidade no horizonte AB.

No caso dos horizontes $A_{p}$ e $A_{2}$, não-coesos, a redução na porosidade total foi causada, provavel mente, por compactação em decorrência da ação das máquinas agrícolas e pisoteio de animais, comuns na área. No horizonte $A B$, a menor porosidade total e macroporosidade devem-se à marcante coesão verificada quando da análise morfológica do perfil.
Após a saturação do sol o para a determinação da capacidade de campo "in si tu", foram quantificadas as variações da umidade do solo em função do tempo, nos horizontes do perfil. As respectivas equações, ajustadas ao modelo cúbico, podem ser vistas na figura 1. Percebe-se claramente a tendência de estabilização da umidade do solo em todos os horizontes, a partir das $72 \mathrm{~h}$ após a saturação do solo, indicando que o movimento descendente da água decresceu sensivelmente. Tomando por baseas considerações de Veihmeyer \& Hendrickson $(1931,1949)$, admitiu-se que o solo havia atingido a capacidade de campo após 72 h de drenagem livre.

Os coeficientes das equações ajustadas ao modelo de van Genuchten, para cada curva de retenção de água dos horizontes do perfil, encontram-se no quadro 2. Utilizando essas equações, determinou-se o potencial matricial da água no sol o correspondente à umidade na capacidade de campo "in situ" (Quadro 3).

No quadro 3, verifica-se que as umidades do solo correspondentes aos potenciais matriciais de -10 e -33 kPa não representam devidamentea estimativa da capacidade de campo do perfil do Latossolo Amarelo. Conforme Reichardt (1975), os valores obtidos em laboratório nunca poderão representar a capaci dade de campo, pois esse critério é estático, enquanto o processo de redistribuição da água no solo é essencialmente dinâmico. Por isso, e por encontrar discrepâncias entre as quantidades de água disponível obtidas em laboratório com aquelas medidas no campo, é que Brunini et al. (1976) recomendam a determinação da capacidade de campo "in situ". Para Reichardt (1988), a procura de um valor de potencial matricial da água no sol o que corresponda à capacidade de campo não tem respaldo teórico. Souza \& Reichardt (1996) complementam afirmando que os resultados obtidos em condições de laboratório são estimativas da retenção de água e não da capacidade de campo.

Quadro 1. Composição textural, distribuição do tamanho dos poros, densidades de partículas e do solo nos horizontes do perfil do Latossolo Amarelo

\begin{tabular}{|c|c|c|c|c|c|c|}
\hline \multirow{2}{*}{\multicolumn{2}{|c|}{ Horizonte Composição textural }} & \multirow{2}{*}{$\begin{array}{l}\text { Porosi dade } \\
\text { total }\end{array}$} & \multirow{2}{*}{\multicolumn{2}{|c|}{ Macroporosidade Microporosidade }} & \multicolumn{2}{|c|}{ Densidade } \\
\hline & & & & & \multirow{2}{*}{\multicolumn{2}{|c|}{$\begin{array}{l}\text { Partícula Solo } \\
\mathrm{kg} \mathrm{dm}^{-3}\end{array}$}} \\
\hline & & \multicolumn{3}{|c|}{$\mathrm{m}^{3} \mathrm{~m}^{-3}$} & & \\
\hline$A_{p}$ & Areia franca & 0,34 & 0,16 & 0,18 & 2,63 & 1,73 \\
\hline $\mathrm{A}_{2}$ & Franco-arenoso & 0,35 & 0,14 & 0,21 & 2,63 & 1,71 \\
\hline$A B$ & Franco-argilo-arenoso & 0,37 & 0,15 & 0,22 & 2,61 & 1,64 \\
\hline $\mathrm{BA}$ & Franco-argilo-arenoso & 0,42 & 0,18 & 0,24 & 2,62 & 1,55 \\
\hline $\mathrm{B}_{\mathrm{w} 1}$ & Franco-argilo-arenoso & 0,42 & 0,18 & 0,24 & 2,65 & 1,53 \\
\hline
\end{tabular}

Média de quatro repetições. 


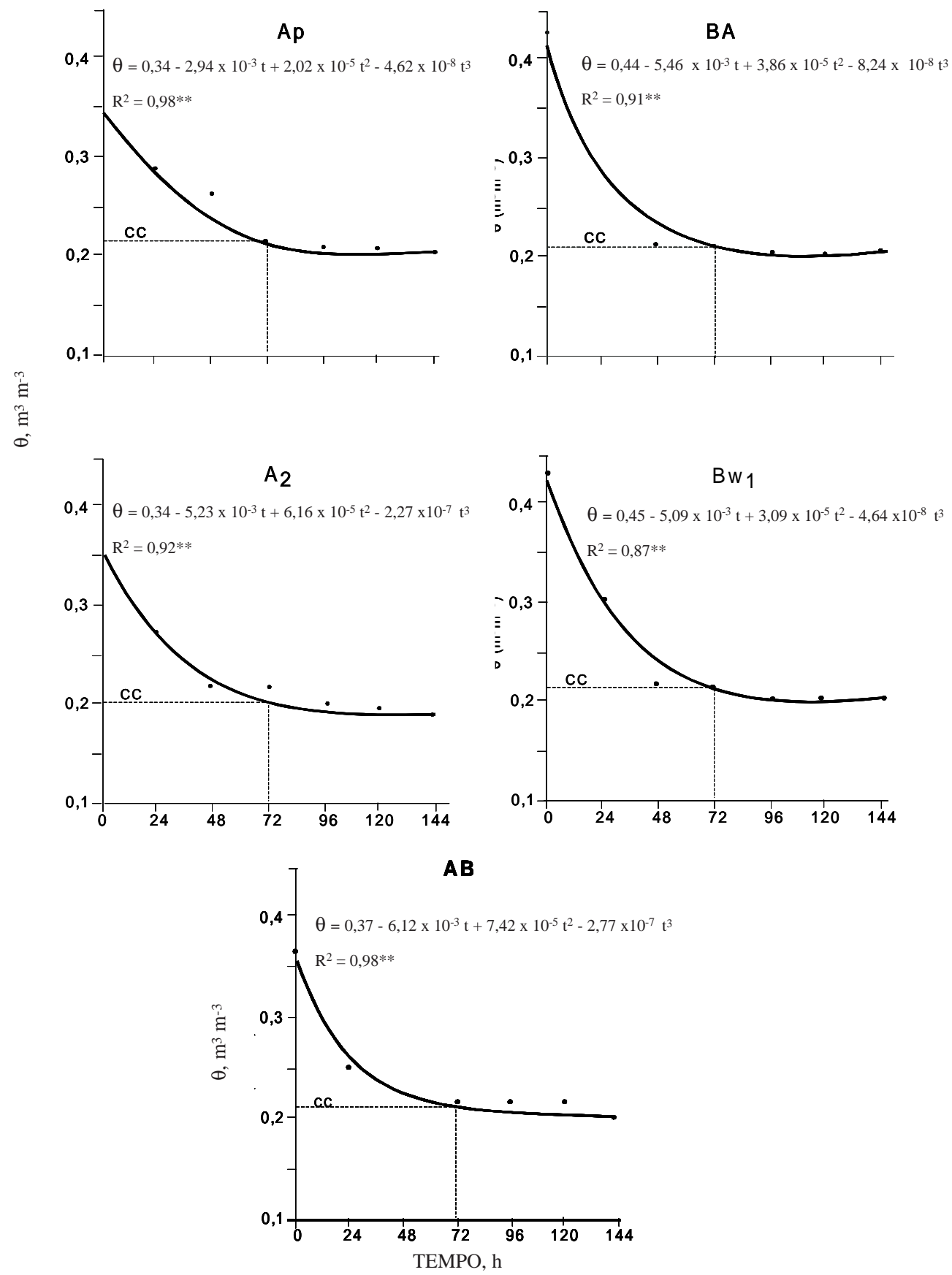

Figura 1. Variação da umidade do solo $(\theta), \mathrm{em} \mathrm{m}^{3} \mathrm{~m}^{-3}$, em função do tempo, em $\mathrm{h}$, para os cinco horizontes do solo e interpolação por polinômio de terceiro grau (CC =Capacidade de campo). ** significativo a $1 \%$.

A figura 2 mostra o perfil de umidade no solo. Analisando o comportamento das curvas 1,2 e 4, nota-se um decréscimo na retenção da água nos horizontes $A_{p}$ e $A_{2}$, quando comparados com os outros, fato perfeitamente compreensível quando se leva em conta a classe textural desses horizontes superficiais (Quadro 1). Entretanto, analisando a curva 3, representante da retenção da água nas condições de campo, verifica-sequeela épraticamente vertical aolongo do perfil. Tal comportamento revela que a água está sendo acumulada na camada de 0-22 am de profundidade, conforme podeser verificado pel o aumento da diferença de umidade do sol o entre a capacidade de campo e o potencial matricial de $-1.500 \mathrm{kPa}$ no quadro 3. Assim, verifica-se, nos horizontes $A_{p}$ e $A_{2}$, um aumento de sua capacidade de campo pelo fato de os horizontes coesos subjacentes dificultarem o processo de drenagem da 
Quadro 2. Umidade residual e de saturação do solo, coeficientes das equações ajustadas ao modelo de van Genuchten (1980) para cada curva de retenção de água dos horizontes do perfil do Latossolo Amarelo coeso

\begin{tabular}{|c|c|c|c|c|c|c|}
\hline \multirow{2}{*}{ Horizonte } & \multicolumn{2}{|c|}{ Umidade } & \multicolumn{3}{|c|}{ Coeficiente } & \multirow{2}{*}{ Coeficiente de ajuste } \\
\hline & Residual & Saturação do solo & $\mathbf{a}$ & $\mathbf{n}$ & b & \\
\hline & \multicolumn{2}{|c|}{$m^{3} m^{-3}$} & & & & \\
\hline$A_{p}$ & 0,068 & 0,342 & 0,992 & 1,121 & 0,433 & 0,999 \\
\hline$A_{2}$ & 0,078 & 0,350 & 1,011 & 2,071 & 0,169 & 0,998 \\
\hline$A B$ & 0,127 & 0,376 & 0,232 & 1,108 & 0,568 & 0,998 \\
\hline B A & 0,112 & 0,408 & 0,417 & 1,122 & 0,491 & 0,999 \\
\hline $\mathrm{B}_{\mathrm{w} 1}$ & 0,119 & 0,423 & 0,488 & 1,951 & 0,299 & 0,999 \\
\hline
\end{tabular}

Quadro 3. Relações entre a umidade do solo na capacidade de campo determinada "ín situ" e os seus correspondentes potenciais matriciais da água no solo, bem como sua diferença em relação à umidade do solo em equilíbrio com o ponto de murcha permanente (potencial matricial de $-\mathbf{1 . 5 0 0} \mathbf{~ k P a}$ )

\begin{tabular}{cccc}
\hline Horizonte & $\begin{array}{c}\text { Capacidade de campo } \\
\text { “in situ” }\end{array}$ & Potencial matricial (- kPa) & $\begin{array}{c}\text { Capacidade de campo “in situ” - } \\
\text { ponto de murcha permanente }\end{array}$ \\
\hline & $\mathrm{m}^{3} \mathrm{~m}^{-3}$ & & $\mathrm{~m}^{3} \mathrm{~m}^{-3}$ \\
$A_{p}$ & 0,209 & 3,0 & 0,134 \\
$A_{2}$ & 0,214 & 7,0 & 0,123 \\
$A B$ & 0,216 & 19,0 & 0,088 \\
$B A$ & 0,205 & 18,0 & 0,085 \\
$B_{w 1}$ & 0,213 & 15,0 & 0,089 \\
\hline
\end{tabular}

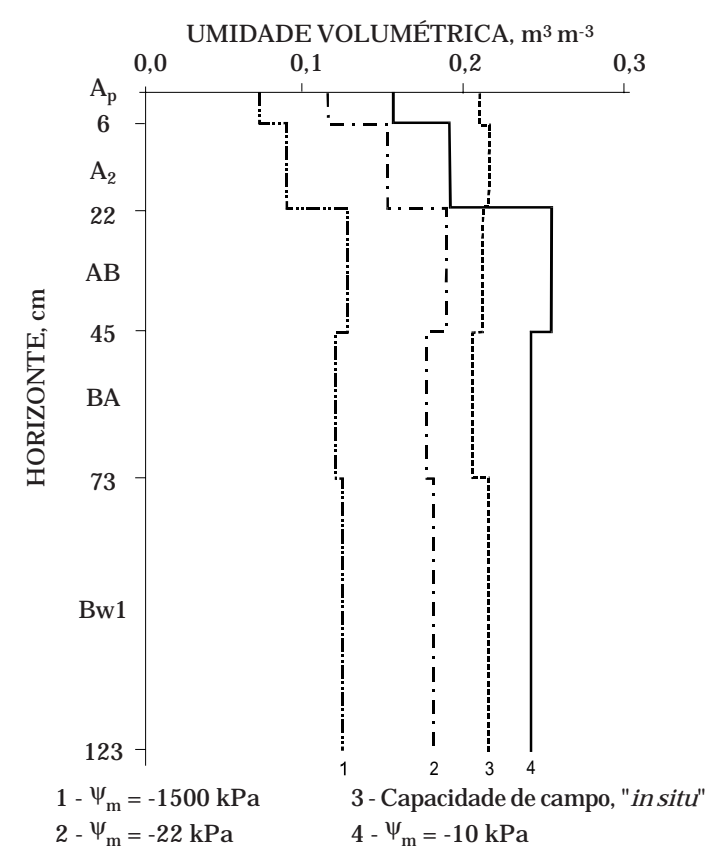

Figura 2. Perfil de umidade do solo após aplicação dos potenciais matriciais indicados (amostras de solo deformadas - curvas 1,2 e 4) e umidade do solo na capacidade de campo determinada "in situ". água (Bernardo, 1986). Tal resultado também corrobora com as ponderações de Reichardt (1975), que afirma que a presença de camadas limitantes ao fluxo, em qualquer posição do perfil, retardará a saída deágua em qual quer das camadas acima delas. Diante do exposto, pode-se inferir que o conceito clássico de capacidade de campo (Veihmeyer \& Hendrickson, 1931,1949) não se aplica ao solo estudado dada sua baixa permeabilidade à água.

Neste caso, visando à aplicação desses conceitos em projetos de irrigação, podem-seindicar os valores da pressuposta capacidade de campo "in situ" unicamente para os horizontes $A B, B A$ e $B_{w 1}$, uma vez que os valores obtidos na determinação em campo para os horizontes $A_{p}$ e $A_{2}$ não expressam sua real capacidade de retenção. Para esses horizontes, em razão das suas texturas arenosas, recomenda-se o uso do potencial matricial da água nosolo de-10 kPa como estimativa dolimite superior de água disponível dessas camadas.

No quadro 4, encontra-se o resumo das informações sobre ponto de murcha permanente, "capaci dade de campo" e seus $\psi_{m}$ correspondentes e faixa de água disponível, indicadas para os horizontes do Latossolo Amarel o estudado. 
Quadro 4. Valores de ponto de murcha permanente, capacidade de campo, potencial matricial da água no solo e faixa de água disponível recomendados para o Latossolo Amarelo

\begin{tabular}{|c|c|c|c|c|}
\hline \multirow{3}{*}{ Horizonte } & \multicolumn{3}{|c|}{ Umidade do solo } & \multirow{3}{*}{ Água disponível } \\
\hline & \multirow{2}{*}{$\begin{array}{l}\text { Ponto de murcha permanente } \\
\qquad\left(\psi_{\mathrm{m}}=-1500 \mathrm{kPa}\right)\end{array}$} & \multicolumn{2}{|c|}{ Capacidade de campo } & \\
\hline & & $\psi_{m}(-k P a)$ & $\theta$ & \\
\hline & $m^{3} m^{-3}$ & & 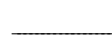 & $n^{-3}$ \\
\hline$A_{p}$ & 0,075 & 10 & 0,157 & 0,082 \\
\hline$A_{2}$ & 0,091 & 10 & 0,198 & 0,107 \\
\hline$A B$ & 0,128 & 19 & 0,216 & 0,088 \\
\hline BA & 0,120 & 18 & 0,205 & 0,085 \\
\hline $\mathrm{B}_{\mathrm{w} 1}$ & 0,124 & 15 & 0,213 & 0,089 \\
\hline
\end{tabular}

\section{CONCLUSÃO}

Tendo em vista que o sol o estudado apresenta os horizontes $A B$ e BA coesos, constituindo forte impedimento mecânico à livre movimentação da água, o conceito clássico de capacidade de campo não se aplica ao solo em questão.

\section{LITERATURA CITADA}

AHUJ A, L.R. \& NIELSEN, D.R. Field soil - water relations. In: STEWART, B.A. \& NIELSEN, D.R., eds. Irrigation of agricultural crops. Madison, American Society of Agronomy, 1990, p.143-189.

BERNARDO, S. Manual deirrigação. 4.ed. Viçosa, UFV, 1986. 486p.

BORGES, E.A. Correlação entre capacidade de campo determinada "in situ" e através de métodos de laboratório. Areia, Universidade Federal da Paraíba, 1979. 79p. (Tese de Mestrado)

BRUNINI, O.; REICHARDT, K. \& GROHMANN , F. Determinação da água disponível em Latossolo Roxo em condições de campo. In: CONGRESSO BRASILEIRO DE CIÊNCIA DO SOLO, 15., Campinas, 1975. Anais. Campinas, Sociedade Brasileira de Ciência do Solo, 1976. p.82-86.

DOURADO NETO, D.; LIER, Q.J .V.; BOTREL, T.A. \& LIBARDI, P.L. Programa para confecção da água no solo utilizando o modelo de Genuchten. Eng. Rural, 1:93-101, 1990.

EMPRESA BRASILEIRA DE PESQUISA AGROPECUÁRIA EMBRAPA. Serviço Nacional de Levantamento e Conservação de Solos. Manual de métodos de análise de solos. Rio de J aneiro, 1979. não paginado.

EMPRESA BRASILEIRA DE PESQUISA AGROPECUÁRIA EMBRAPA. Sistema brasileiro de classificação de solos. Brasília, Embrapa Solos, 1999. 412p.

FERNANDES, B. \& SYKES, D.J . Capacidade de campo e a retenção de água em três solos de Minas Gerais. R. Ceres, 15:1-37, 1968.

FERREIRA, M.M. \& MARCOS, Z.Z. Estimativa da capacidade de campo em Latossolo Roxo distrófico e regossolo através do ponto de inflexão da curva característica de umidade. Ci. Prat., 7:96-101, 1983.
FREIRE, J .C. Condutividade hidráulica e capacidade de campo de Latossolo Roxo distrófico não saturado. R. Bras. Ci. Solo, 3:73-77, 1979.

GENUCHTEN, M.Th. van. A closed - form for predicting the hydraulic conductivity of unsatured soils. Soil Sci. Soc. Am. J ., 44:892-898, 1980.

J AMISON, V.C. Changes in air water relationships due to structural improvement of soils. Soil Sci., 76:143-151, 1953.

KIEHL, E.J . Manual de edafologia. São Paulo, Ceres, 1979. 264p.

MACLEAN, A.H. \& YAGER, T.V. Available water capacity of Zambian soils in relation to pressure plate measurements and particle size analyses. Soil Sci., 13:23-29, 1972.

MARCOS, Z.Z. Morphologic and physical properties of finetextured oxissols state of São Paulo. Ohio, Ohio State University, 1971. 272p. (Tese de Doutorado)

MEDINA, F.B. \& OLIVEIRA J r., R. Relações entre capacidade de campo determinada "in situ" e em laboratório em Latossolo Amarel o muito argiloso. R. Bras. Ci. Solo, 11:9195, 1987.

OLIVEIRA, J .B.; J ACOMINE, P.K. \& CAMARGO, M.N. Classes gerais de sol os do Brasil. J aboticabal, FUNEP, 1992. 201p.

REICHARDT, K. Processos de transferência no sistema soloplanta-atmosfera. 3.ed. Piracicaba, USP/Cargill, 1975. 286p.

REICHARDT, K. Capacidade decampo. R. Bras. Ci. Solo, 12:211216, 1988.

RICHARDS, L.A. Methods of measuring moisture tension. Soil Sci, 58:95-112, 1949.

SALTER, P.J . \& HAWORTH, F. The available- water capacity of a sandy loam soil. I. A critical comparison of methods of determining the moisture content of soil at field capacity and the permanent wilting percentage. J . Soil Sci., 12:326334, 1961

SOUZA, L.D. \& REICHARDT, K. Estimativas e utilização da capacidade de campo. R. Bras. Ci. Solo, 20:183-189, 1996.

VEIMEHYER, F.J . \& HENDRICKSON, A.H. The moisture equivalent as a measure of the field capacity of soils. Soil Sci., 32:181-193, 1931.

VEIMEHYER, F.J. \& HENDRICKSON, A.H. Methods of measuring field capacity and permanent wilting percentage of soils. Soil Sci., 68:75-94, 1949. 